\title{
Structural transition in alcohol-water binary mixtures: A spectroscopic study
}

\author{
TUHIN PRADHAN, PIUE GHOSHAL and RANJIT BISWAS* \\ Department of Chemical, Biological and Macromolecular Sciences, and Unit for Nanoscience and \\ Technology, S. N. Bose National Centre for Basic Sciences, JD Block, Sector III, Salt Lake City, \\ Kolkata 700098 \\ e-mail: ranjit@bose.res.in
}

MS received 18 September 2007; accepted 29 January 2008

\begin{abstract}
The strengthening of the hydrogen bonding (H-bond) network as well as transition from the tetrahedral-like water network to the zigzag chain structure of alcohol upon increasing the alcohol concentration in ethanol-water and tertiary butanol (TBA) - water mixtures have been studied by using both steady state and time resolved spectroscopy. Absorption and emission characteristics of coumarin 153 (C153), a widely used non-reactive solvation probe, have been monitored to investigate the structural transition in these binary mixtures. The effects of the hydrogen bond (H-bond) network with alcohol concentration are revealed by a minimum in the peak frequency of the absorption spectrum of $\mathrm{C} 153$ which occur at alcohol mole fraction $\sim 0.10$ for water-ethanol and at $\sim 0.04$ for water-TBA mixtures. These are the mole fractions around which several thermodynamic properties of these mixtures show anomalous change due to the enhancement of H-bonding network. While the strengthening of $\mathrm{H}$-bond network is revealed by the absorption spectra, the emission characteristics show the typical non-ideal alcohol mole fraction dependence at all concentrations. The time resolved anisotropy decay of C153 has been found to be bi-exponential at all alcohol mole fractions. The sharp change in slopes of average rotational correlation time with alcohol mole fraction indicates the structural transition in the environment around the rotating solute. The changes in slopes occur at mole fraction $\sim 0 \cdot 10$ for TBA-water and at $\sim 0 \cdot 2$ for ethanol-water mixtures, which are believed to reflect alcohol mole fraction induced structural changes in these alcohol-water binary mixtures.
\end{abstract}

Keywords. Alcohol-water binary mixtures; hydrogen-bonding network; structural transition; absorption and fluorescence emission; fluorescence anisotropy.

\section{Introduction}

Alcohol-water binary mixtures have been studied extensively over the years in order to correlate the solution structure with the observed anomaly in several thermodynamic properties of these mixtures. ${ }^{1-31}$ As a subtle balance between the hydrophobic and hydrogen bonding (H-bonding) interactions determines the solution structure and hence the properties, attempts have been made to understand how the hydrophobic interactions among the alkyl groups of the alcohol molecules and the $\mathrm{H}$-bonding interactions among the hydroxyl groups (-OH groups) of water and alcohol molecules assist each other to retain the structure of the pure form of the respective species. Neutron diffraction, ${ }^{7-8,28}$ small angle X-ray scatter-

\footnotetext{
*For correspondence
}

ing (SAXS), ${ }^{18-20}$ light scattering ${ }^{18,32-34}$ and other experimental studies ${ }^{9,12-13}$ have indicated that microscopic phase separation and cluster formation dominate the mixing in alcohol-water systems. While the aggregation of alcohol molecules are often linked to the anomalous change in several thermodynamic properties, modifications in solution structure are believed to be responsible for the observed sharp change in the ultrasound absorption coefficient of alcoholwater binary mixtures. ${ }^{9,35-36}$ Even though several experimental studies support the idea of formation of clathrate type structures between water and alcohol molecules at very low alcohol concentration, neutron diffraction studies with the lowest alcohol mole fraction have not found any evidence for such a structure.

Computer simulation studies ${ }^{22-25}$ with realistic potentials have aided the experiments where the self-association of alcohol molecules and structural 
enhancement are studied by using the surface distribution function (SDF) ${ }^{37}$ approach. Simulation studies with tertiary butanol (TBA) in water have suggested that the life-time of the clusters formed through aggregation of 2-4 alcohol molecules is $\sim 20-30$ ps and undergo continual change inducing fluctuations in the micro-environments. ${ }^{23}$ These studies have also examined the question whether water-cage encapsulates the alcohol molecules or the alcohol hydroxyl groups are incorporated in the water-cage by participating in the H-bonding network while leaving the methyl groups interacting through hydrophobic interaction. Theoretical studies based on the RISM (Reference Interaction Site Model) approach with modified closure relations have indicated strengthening of $\mathrm{H}$ bonding between all species and also explained the non-monotonic alcohol mole fraction dependence of isothermal compressibility. ${ }^{25,38}$ According to these studies, alcohol molecule resides in the cavity of the H-bonding network created by the surrounding water molecules which, in turn, lowers the compressibility of the medium. ${ }^{38}$ However, with further addition of alcohol, the tetrahedral network structure gradually converts to the zigzag chain structure of alcohol increasing the compressibility of the water-alcohol mixtures. ${ }^{38}$ Therefore, the structural enhancement and the mode of accommodating alcohol molecules within the tetrahedral network in the water-rich region have aroused immense interest among the experimentalists and theoreticians alike leading to constant debate and discussion.

Since the structure of the solution is intimately related to its dynamics, ${ }^{39,40}$ structural modification upon addition of alcohol will also affect the solution dynamics. Several dielectric relaxation studies have addressed this question and also the non-ideality in such binary mixtures. ${ }^{12,21}$ It is known that the interdiffusion and preferential solvation play an important role in determining the time scale of environment reorganization around an excited dipolar solute or an ion in these dipolar mixtures..$^{39-51}$ Therefore, for simple chemical events such as ion diffusion and charge transfer reactions, substantial effects are expected from the modifications in both the structure and dynamics of these water-alcohol mixtures. Note that neutron diffraction studies of cyclohexenewater-alcohol ternary mixtures ${ }^{52}$ have suggested much less heterogeneity in solution structure and hence, the size mediated coulomb interactions are expected to dominate the solution structure and nonideality in presence of bigger particle (as third com- ponent). Recent theoretical studies based on the mean spherical approximation (MSA) have also indicated that the size-mediated interactions can indeed semi-quantitatively describe the non-ideality for larger ions in several complex mixtures, such as watermethanol, water-ethanol, water-tertiary butanol and dimethyl sulfoxide (DMSO)-acetonitrile mixtures. ${ }^{53-54}$

In this article, we have studied the concentration dependent structural transitions in alcohol-water binary mixtures at room temperature by following the steady state absorption and emission properties of a non-reactive probe at different alcohol mole fractions. In addition, time resolved fluorescence anisotropy has been investigated to look at the solutesolvent coupling at the dynamic level. $\mathrm{C} 153$ has been chosen as a non-reactive probe for our study since experimental studies and quantum mechanical calculations have indicated $\mathrm{C} 153$ as one of the best solvation probes normally used in such studies ${ }^{55,56}$ Ethanol and tertiary butanol (TBA) have been chosen since these alcohols are fully miscible with water at any proportion and at any temperature. In addition, TBA is the most hydrophobic alcohol to remain soluble with water without any phase boundary. More importantly, aqueous binary mixtures of these alcohols show well-structured peak when several thermodynamic properties are followed as a function of alcohol mole fraction. ${ }^{1-5}$ Also, the values of the mole fraction at which the above-mentioned peak occurs vary sharply with the identity of the alcohol in the mixture. This probably reflects the differing ability of these alcohols to perturb the water structure through hydrophobic interactions that depends significantly on the bulkiness of the alkyl groups attached to a particular alcohol molecule.

The main results of the paper are as follows. The peak frequency of absorption spectrum of $\mathrm{C} 153$ shows a minimum with the alcohol mole fraction for both ethanol and TBA in binary aqueous mixtures. While the minimum for ethanol-water mixture occurs at mole fraction $\sim 0 \cdot 10$, it is $\sim 0.04$ for TBA-water mixture. Interestingly, emission peak frequencies do not exhibit such minima and show only the nonideality in average polarity. Transition moments, quantum yields, radiative and non-radiative rates show, on an average, non-ideal alcohol mole fraction dependence. Time dependent fluorescence anisotropy decay of C153 have been found to be bi-exponential at all alcohol mole fractions with well-separated time scales. The average rotational correlation time, when plotted as a function of alcohol concentration, 
show a change in slope at the alcohol mole fractions where structural transitions from tetrahedral network to chain structure are expected to occur. The biexponential nature of the anisotropy decay indicates aggregation of alcohol molecules is confined within a narrow distribution of the number in alcohol association.

The rest of the paper is organized as follows. Experimental details are given in the next section. Section 3 contains the results and discussion. The concluding remarks are provided in $\S 4$.

\section{Experimental}

C153 was obtained from Exciton and used as received. Tertiary butanol (TBA) were purchased from Sigma-Aldrich, USA and Ethanol from SRL, India. Both these alcohols were of the highest grade and used without further purification. De-ionised water (Millipore) was used to prepare the aqueous solutions of these alcohols. Caution was exercised to ensure the accuracy of the mole fraction, particularly near the very low alcohol and water concentrations. Subsequently, a small grain of $\mathrm{C} 153$ was dissolved in a small volume of the stock solution taken in a quartz cuvette of optical path length $1 \mathrm{~cm}$ and stirred the solution for some time to ensure complete dissolution. Absorption spectra were then recorded (Model UV-2450, SHIMADZU) for solutions containing different concentrations of alcohols spanning the entire range. The emission spectra were recorded (SPEX Fluoromax-3, Jobin-Yvon, Horiba) after adjusting the absorbance of the solution to $0 \cdot 1$ or less with excitation wavelength fixed at $409 \mathrm{~nm}$. Solvent blanks were subtracted from both the absorption and emission spectra prior to analysis and converted to frequency representation after properly weighting the emission intensity with $\lambda^{2}$. Note that bubbling few samples with dry argon gas showed very little or no effects and hence most of the samples were not deoxygenated.

Absorption and emission peak frequencies were calculated by simply averaging the numbers obtained from fitting the upper half of the spectrum with an inverted parabola, first moment and the arithmetic mean of the frequencies at half intensities on both blue and red ends of each of the spectrum. ${ }^{57,58}$ The consistency of the above method was further checked by fitting the absorption (emission) spectra to a log-normal function by broadening and shifting the absorption (emission) spectrum of $\mathrm{C} 153$ in a non- polar solvent. ${ }^{59,60}$ This was necessary particularly when the spectra were noisy.

Time resolved fluorescence ansitropy decays were collected using time correlated single photon counting (TCSPC) technique based on a laser system (Lifespec-ps, Edinburgh, UK) with $409 \mathrm{~nm}$ light as excitation. The full width at half maximum of the instrument response function (IRF) with the above excitation was approximately $75 \mathrm{ps}$. The emission decays were collected with an emission band pass of $8 \mathrm{~nm}$. Emission decays with emission polarizer at magic angle were collected to obtain the average lifetime of $\mathrm{C} 153$ at different alcohol mole fractions in these mixtures. Subsequently, the collected emission decays were de-convoluted from the IRF and fitted to multi-exponential function using an iterative re-convolution algorithm. ${ }^{61}$ Such fitting enables one to capture dynamical events with time constant as fast as $\sim 15 \mathrm{ps}$ with reasonable accuracy ${ }^{61}$ All the experiments were performed at room temperature, $295 \pm 0 \cdot 5 \mathrm{~K}$.

Emission decays for anisotropy were collected at the peak wavelength of the steady state emission bands so that the effects of fast decay or rise due to solvent reorganization is minimized. ${ }^{62}$ For a few cases, emission decays were collected at two or three different wavelengths around the peak of the emission band and the analysed data were found to vary within a small uncertainty. Time resolved fluorescence anisotropies, $r(t)$, were calculated from the collected and back ground subtracted parallel $\left(I_{\|}(t)\right)$ and perpendicular $\left(I_{\perp}(t)\right)$ decays by using the following well-known formula ${ }^{62}$

$$
r(t)=\frac{I_{\|}(t)-G I_{\perp}(t)}{I_{\|}(t)+2 G I_{\perp}},
$$

where $G$ accounts for the differential sensitivity to the two polarizations which was obtained by tail matching the intensity decays $I_{\|}(t)$ and $I_{\perp}(t)$. The average value for $G$ obtained by tail matching the relevant decays at times longer than the anticipated rotation time is $1 \cdot 15 \pm 0 \cdot 05$.

The time resolved anisotropy constructed from the collected emission decays by using (1) then fitted to a bi-exponential function after de-convoluting from the IRF using an iterative re-convolution fitting program. The following form of the bi-exponential function was used for this purpose ${ }^{62}$

$$
r(t)=r(0)\left[a_{1} \exp \left(-t / \tau_{1}\right)+\left(1-a_{1}\right) \exp \left(-t / \tau_{2}\right)\right],
$$


where $\tau_{1}$ and $\tau_{2}$ represent the time constants associated with the decay components constituting the $r(t)$. $r(0)$ denotes the initial anisotropy and was taken as 0.376 for fitting the time resolved anisotropies of C153 in all the mixtures studied here. ${ }^{62}$ The alcohol mole fraction dependence of rotational motion of C153 in these binary polar mixtures is presented in terms of the average rotational correlation time, $\left\langle\tau_{\text {rot }}\right\rangle=a_{1} \tau_{1}+\left(1-a_{1}\right) \tau_{2}$, which comes from the time integration of (2) after normalizing with $r(0)$.

\section{Results and discussion}

\subsection{Steady state studies}

Absorption and emission spectra of C153 in TBAwater solutions at $0.035,0.1,0.5$ TBA mole fractions as well as those in pure water and TBA are shown in figure 1. These TBA mole fractions are cho-

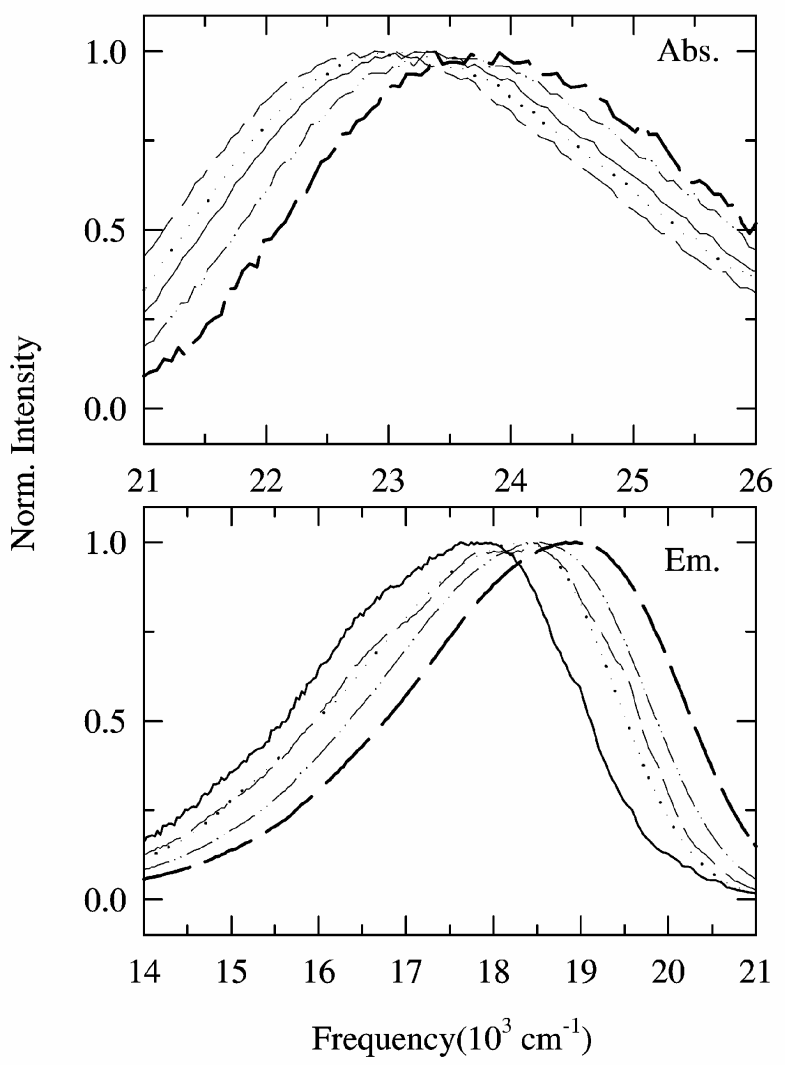

Figure 1. Absorption (upper panel) and emission (lower panel) spectra of C153 in different mole fractions of tertiary butyl alcohol (TBA) - water mixture. Spectra shown here correspond to the following mole fractions of TBA: pure water (solid line), 0.035 (long dash, thin), $0 \cdot 1$ (dotted), 0.5 (dash-dot-dot), pure TBA (short dash, thick). sen so that the effects of strengthening of H-bond network and gradual change in $\mathrm{H}$-bonding structure (from tetrahedral-like network at low concentration to zigzag chain at higher mole fractions) on the stabilization of ground and excited states of a fluorescent probe are represented. As already mentioned, aqueous TBA solutions with low TBA mole fractions are characterized by aggregation of TBA molecules via interactions among hydrophobic tertiary butyl $\left(-\mathrm{CMe}_{3}, \mathrm{Me}=\mathrm{CH}_{3}\right)$ groups, whereas at extremely dilute solution alcohol molecules induce strengthening of $\mathrm{H}$-bonds among all species. In concentrated TBA solution, H-bonding chain structure similar to that in pure alcohol persists. While 0.04 TBA mole fraction corresponds to the maximum anomaly in several thermodynamic properties of aqueous TBA solution, ${ }^{1-5}$ transition from the TBATBA intermolecular contact to the TBA-water molecular association is believed to occur at $\sim 0.1$ TBA mole fraction. ${ }^{6-7,18}$ For ethanol-water mixtures, the structural transition is reflected at ethanol mole fraction $\sim 0 \cdot 20$, whereas the maximum anomaly occurs at $\sim 0.10$ mole fraction of ethanol. ${ }^{18}$

One of the most interesting aspects of figure 1 is that at low alcohol mole fractions (up to $\sim 0.04$ for TBA and $\sim 0 \cdot 10$ for ethanol), absorption spectrum of C153 shows a red shift with the increase in alcohol concentration, whereas the emission spectrum shifts towards blue. At higher alcohol concentrations, both absorption and emission spectra show blue shift as the average polarity of the medium decreases upon successive addition of alcohol. The alcohol mole fraction dependence of the peak frequency of absorption and emission spectra and spectral bandwidths (full width at half maximum) for $\mathrm{C} 153$ in the aqueous solutions of these two alcohols have been studied in detail and are shown in figure 2. It is evident from this figure that except at dilute alcohol concentration, the absorption spectra of C153 in aqueous solutions of ethanol and TBA show a nonideal alcohol mole fraction dependence. The emission spectra, on the other hand, exhibit a continuous blue shift with TBA mole fraction and reveal the typical non-ideality in average polarity of such alcohol-water mixtures. For the entire alcohol concentration range the total blue shift for the emission band in TBA-water mixture is $\sim 1500 \mathrm{~cm}^{-1}$, whereas it is $\sim 900 \mathrm{~cm}^{-1}$ for ethanol-water mixture. This reflects the difference in static dielectric constants $\left(\varepsilon_{0}\right)$ of these two alcohols (24 for ethanol and $\sim 12$ for TBA) ${ }^{10,11}$ Perhaps the most interesting feature of this figure is the red-shift of the absorption spectrum 
by about $1000 \mathrm{~cm}^{-1}$ while going from the infinitely dilute TBA-water solution to 0.04 TBA mole fraction. For ethanol-water mixture, the red shift continues up to $0 \cdot 10$ mole fraction and it is much less $\left(\sim 500 \mathrm{~cm}^{-1}\right)$ than that for TBA.

Since addition of alcohol in water facilitates formation of alcohol clusters and enhances the H-bonding between all species, the microheterogeneity and the related structural modifications will affect the spectral properties of a dissolved solute. The enhanced $\mathrm{H}$-bonding structure at low TBA concentrations is probed by a recent study on compressibility of TBAwater mixtures using the RISM theory ${ }^{38}$ where realistic potentials have been used to represent both the species. ${ }^{25,38}$ This theoretical study and comparisons with relevant experimental data have suggested that at low TBA concentration the cavity in the H-bonding network of water is occupied by the alkyl groups of the clustered TBA molecules and thereby reducing the compressibility of the solution. ${ }^{38}$ Therefore, the reduced compressibility is an indicative of a more compact solvation environment surrounding the probe (C153) molecule. This increased compactness due to the enhancement of local structure then naturally stabilizes the energy levels of the probe, leading to the observed red shift in the absorption spectrum. However, with further addition of TBA, tetrahedral

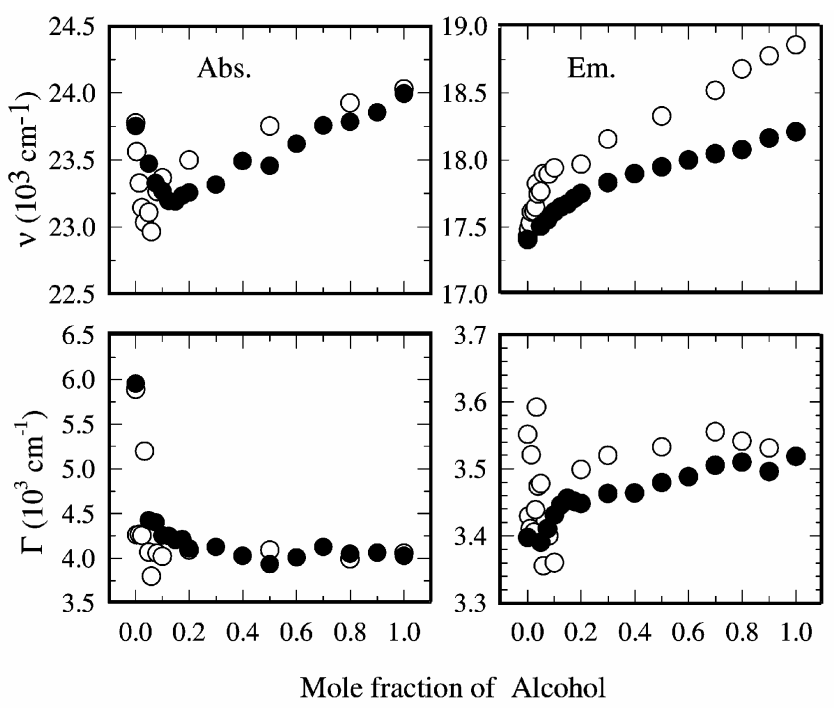

Figure 2. Alcohol mole fraction dependence of absorption ( $v_{\mathrm{abs}}$, left panel) and emission $\left(v_{\mathrm{em}}\right.$, right panel) peak frequencies and line widths (full width at half maxima, $\Gamma$ ) of the absorption spectra and emission spectra of $\mathrm{C} 153$ in TBA-water (open circles) and ethanol-water (filled circles) mixtures. The estimated uncertainty in frequencies and widths is $\pm 300 \mathrm{~cm}^{-1}$.
H-bonding network structure of water-rich solutions gradually converts to the chain-like alcohol structure at concentrated TBA solutions. ${ }^{25}$ Several studies ${ }^{1-2,7,25}$ of TBA-water systems have revealed that such a structural transition occurs at TBA mole fraction $\sim 0 \cdot 10$, a value at which the non-ideality sets in. The corresponding mole fraction for ethanol-water mixture is $\sim 0 \cdot 2$. However, the question is then why emission frequency does not exhibit such a sharp TBA mole fraction dependence? Simulation studies of TBA-water solutions have indicated that even though clusters of three or four TBA molecules are formed but undergo continual change on time scales of tens of picoseconds. ${ }^{23}$ Since the stability times of these clusters $(20-30 \mathrm{ps})^{23}$ are many times smaller than the average life time of the excited state $(>1 \mathrm{~ns})$ of the probe, the solvent environment surrounding the photo-excited probe undergoes a large number of relatively rapid fluctuations. These environmental fluctuations might average out the subtle structural modification in dilute aqueous solutions of TBA allowing the solution polarity to primarily govern the emission transition energy.

Small angle X-ray scattering studies ${ }^{17-20}$ of alcohol-water mixtures have revealed that the heterogeneity is much less in ethanol-water solutions than that in TBA-water mixtures. In addition, $a b$ initio calculations for gas phase dimers suggest that the hydrogen bonding energy between two ethanol molecules is nearly the same as that between a water-water and water-ethanol molecules. ${ }^{63,64}$ Moreover, unlike the case for TBA-water mixtures, the hydrophobic contribution is smaller for ethanol-water mixtures and hence formation of stable water cage around an ethanol molecule is less likely. As a result, the solution heterogeneity and its effects are much less pronounced for ethanol-water than that in TBA-water mixtures. This 'increased' homogeneity is also responsible for the weaker non-ideality observed for the absorption peak frequencies of $\mathrm{C} 153$ in ethanolwater solutions.

Another interesting aspect of figure 2 is the TBA mole fraction dependence of absorption and emission bandwidths of $\mathrm{C} 153$. The absorption bandwidth (bottom-left panel) shows a sharp decrease with TBA mole fraction up to $\sim 0.04$. However, the dependence of absorption bandwidth on TBA mole fraction is weaker at mole fractions greater than 0.04 and the total decrease in bandwidth in this range is only about $200 \mathrm{~cm}^{-1}$ which is comparable to frequency shift versus bandwidth behaviour observed earlier in neat solvents. ${ }^{55}$ The narrowing of absorption spectrum 
of $\mathrm{C} 153$ by $\sim 1000 \mathrm{~cm}^{-1}$ upon increasing the TBA concentration to $\sim 0.04$ mole fraction is anomalously large and apparently contradicts the observations made in earlier studies. ${ }^{55}$ As the spectral width in a binary mixture derives contributions from both the probe-solvent interaction strength and the heterogeneity in the surrounding solvent environment due to both density and concentration fluctuations, the narrowing of absorption spectrum may be regarded as a reflection of a novel interplay between the lowering of polarity and the loss of microscopic heterogeneity on each successive addition of TBA in water. These arguments might also explain the narrowing of absorption bands for ethanol-water mixtures up to ethanol mole fraction $\sim 0 \cdot 10$. For emission, however, a decrease in bandwidth is observed for very dilute TBA concentration (up to $\sim 0.04$ mole fraction) and then increases with further addition of TBA. Unfortunately, the decrease in bandwidth is only $\sim 300 \mathrm{~cm}^{-1}$, which is comparable to the instrumental resolution and hence no definite conclusion could be made. Note that the broadening of the emission band with simultaneous blue shift in TBA mole fractions $>0.04$ is similar to what has been observed earlier with $\mathrm{C} 153$ in pure solvents. ${ }^{55}$ In a recent study with $\mathrm{C} 153$ in hexane-heptanol binary mixtures, ${ }^{41}$ the emission bandwidth is found to increase up to alcohol mole fraction $\sim 0 \cdot 10$ and then decreases with further addition of alcohol. This has been explained in terms of heterogeneity of solution structure. ${ }^{41}$ However, no information is available in this study regarding the correlation between the red shift of the absorption spectrum and the absorption bandwidth. This is, therefore, one of the new results from the steady state spectroscopic studies of alcohol-water binary mixtures reported here.

We have measured quantum yield, radiative and non-radiative rates, and transition moments of $\mathrm{C} 153$ in aqueous solutions of ethanol and TBA in order to investigate the effects of solution structure on these quantities. Quantum yield has been determined by using the following relation ${ }^{59,61}$

$$
\phi_{S}=\phi_{R}\left(\frac{n S^{2}}{n R^{2}}\right)\left(\frac{I_{S}}{I_{R}}\right)\left(\frac{1-10^{-0.5 A_{R}}}{1-10^{-0.5 A_{S}}}\right) .
$$

Quinine sulphate dihydrate in $0.05 \mathrm{M} \mathrm{H}_{2} \mathrm{SO}_{4}$ has been used as reference $\left(\phi_{R}=0.508\right){ }^{61}$ In (3), $n_{x}$ represents refractive index of the reference solution $(R)$ and sample $(S), I$ the integrated emission intensity, and $A$ the absorbance. Refractive indices of the alcohol-water solutions at different mole fractions of ethanol and TBA have been measured (296.15 \pm $1 \mathrm{~K}$ ) and summarized in table 1. Quantum yields of C153 determined at various mole fractions of these two alcohols are shown in the upper panel of figure 3 . The error bar associated with these values is typically $\pm 10 \%$ of the values reported here for the alcohol mole fraction range $0 \cdot 10-1 \cdot 0$. For alcohol mole fractions lower than $0 \cdot 10$, the error bar is slightly larger $( \pm 15 \%)$ because of the low signal-to-noise ratio in this range. As expected, the alcohol mole fraction dependence of quantum yield is a non-ideal one. Similar alcohol mole fraction dependence is also found in our earlier studies for the quantum yield of a TICT molecule in TBA-water mixtures. ${ }^{59,65}$

The following relation has been used to determine the radiative rate: ${ }^{61} k^{\text {rad }}=\phi \mid\langle\tau\rangle$, where the average lifetime $(\langle\tau\rangle)$ has been calculated by using the relation, ${ }^{61}\langle\tau\rangle=\sum_{i} a_{i} \tau_{i} / \sum_{i} a_{i}$ The amplitudes $\left(a_{i}\right)$ and time constants $\left(\tau_{i}\right)$ are obtained by fitting the relevant magic angle emission decays. These fit parameters are summarized in table 2 . The non-radiative rates $\left(k^{\mathrm{nr}}\right)$ for all alcohol mole fractions have also been calculated by using the following formula: $k^{\mathrm{nr}}=1 /\langle\tau\rangle-k^{\mathrm{rad}}$. The calculated radiative and nonradiative rates for $\mathrm{C} 153$ in ethanol-water and TBA water mixtures at different alcohol mole fractions are shown in the middle panels of figure 3 . The maximum error bar for these radiative rates is $\pm 20 \%$ of the values reported here. It is interesting to note that the radiative rates for $\mathrm{C} 153$ are similar in both ethanol-water and TBA-water mixtures and remains

Table 1. Refractive indices of ethanol-water and TBAwater mixtures at different alcohol mole fractions.

\begin{tabular}{|c|c|c|c|}
\hline $\begin{array}{l}\text { Mole fraction } \\
\text { of EtOH }\end{array}$ & $n_{s}$ & $\begin{array}{l}\text { Mole fraction } \\
\text { of TBA }\end{array}$ & $n_{s}$ \\
\hline 0 & $1 \cdot 331$ & $0 \cdot 0$ & $1 \cdot 331$ \\
\hline 0.05 & $1 \cdot 339$ & $0 \cdot 015$ & $1 \cdot 336$ \\
\hline 0.075 & $1 \cdot 3415$ & 0.035 & $1 \cdot 344$ \\
\hline $0 \cdot 1$ & $1 \cdot 345$ & 0.05 & $1 \cdot 346$ \\
\hline $0 \cdot 125$ & $1 \cdot 348$ & 0.06 & $1 \cdot 35$ \\
\hline $0 \cdot 15$ & $1 \cdot 351$ & 0.08 & $1 \cdot 355$ \\
\hline $0 \cdot 175$ & $1 \cdot 353$ & $0 \cdot 1$ & $1 \cdot 357$ \\
\hline $0 \cdot 2$ & $1 \cdot 355$ & $0 \cdot 2$ & $1 \cdot 368$ \\
\hline $0 \cdot 3$ & $1 \cdot 358$ & 0.5 & $1 \cdot 379$ \\
\hline 0.4 & $1 \cdot 36$ & 0.8 & $1 \cdot 381$ \\
\hline 0.5 & $1 \cdot 361$ & $1 \cdot 0$ & $1 \cdot 382$ \\
\hline 0.6 & $1 \cdot 362$ & & \\
\hline 0.7 & $1 \cdot 362$ & & \\
\hline 0.9 & $1 \cdot 36$ & & \\
\hline $1 \cdot 0$ & $1 \cdot 359$ & & \\
\hline
\end{tabular}


Table 2. Magic angle emission decay fit parameters of C153 in ethanol-water and TBA-water mixtures at different alcohol mole fractions.

\begin{tabular}{|c|c|c|c|c|c|c|c|c|c|}
\hline \multirow{2}{*}{$\begin{array}{l}\text { Mole fraction } \\
\text { of ethanol }\end{array}$} & \multicolumn{4}{|c|}{ Ethanol $^{\mathrm{a}}$} & \multirow{2}{*}{$\begin{array}{l}\text { Mole fraction } \\
\text { of TBA }\end{array}$} & \multicolumn{4}{|c|}{ Tertiary butanol } \\
\hline & $\tau_{1}(\mathrm{ps})$ & $\tau_{2}(\mathrm{~ns})$ & $a_{1}(\%)$ & $a_{2}(\%)$ & & $\tau_{1}(\mathrm{ps})$ & $\tau_{2}(\mathrm{~ns})$ & $a_{1}(\%)$ & $a_{2}(\%)$ \\
\hline 0 & 150 & 1.67 & $6 \cdot 2$ & $93 \cdot 8$ & $0 \cdot 0$ & 128 & 1.68 & $3 \cdot 5$ & $96 \cdot 5$ \\
\hline 0.05 & 84 & $2 \cdot 06$ & $7 \cdot 7$ & $92 \cdot 3$ & 0.015 & 95 & 1.99 & $5 \cdot 0$ & 95.0 \\
\hline 0.075 & 69 & $2 \cdot 26$ & $8 \cdot 9$ & $91 \cdot 1$ & 0.035 & 98 & $2 \cdot 42$ & 8.7 & $91 \cdot 3$ \\
\hline $0 \cdot 1$ & 69 & $2 \cdot 49$ & $10 \cdot 6$ & $89 \cdot 4$ & 0.05 & 116 & $2 \cdot 87$ & $6 \cdot 6$ & $93 \cdot 4$ \\
\hline $0 \cdot 125$ & 88 & $2 \cdot 68$ & 9.7 & $90 \cdot 3$ & 0.06 & 126 & 3.09 & $7 \cdot 6$ & $92 \cdot 4$ \\
\hline $0 \cdot 15$ & 84 & $2 \cdot 85$ & $9 \cdot 3$ & $90 \cdot 7$ & 0.08 & 178 & $3 \cdot 31$ & $5 \cdot 1$ & $94 \cdot 9$ \\
\hline $0 \cdot 175$ & 86 & $2 \cdot 98$ & $10 \cdot 2$ & $89 \cdot 8$ & $0 \cdot 1$ & 173 & $3 \cdot 43$ & $8 \cdot 1$ & 91.9 \\
\hline $0 \cdot 2$ & 120 & 3.05 & $6 \cdot 9$ & $93 \cdot 2$ & $0 \cdot 2$ & 190 & $3 \cdot 75$ & $6 \cdot 5$ & 93.5 \\
\hline $0 \cdot 3$ & 84 & $3 \cdot 38$ & $11 \cdot 6$ & $88 \cdot 4$ & $0 \cdot 5$ & 256 & $4 \cdot 37$ & $5 \cdot 8$ & $94 \cdot 2$ \\
\hline 0.4 & 99 & $3 \cdot 61$ & $9 \cdot 3$ & $90 \cdot 7$ & $0 \cdot 8$ & 245 & $4 \cdot 90$ & 8.9 & $91 \cdot 1$ \\
\hline $0 \cdot 5$ & 79 & $3 \cdot 80$ & $11 \cdot 1$ & $88 \cdot 9$ & $1 \cdot 0$ & 224 & $5 \cdot 20$ & $10 \cdot 6$ & $89 \cdot 4$ \\
\hline 0.6 & 91 & $3 \cdot 96$ & $9 \cdot 2$ & $90 \cdot 8$ & & & & & \\
\hline $0 \cdot 7$ & 79 & $4 \cdot 15$ & $11 \cdot 5$ & $88 \cdot 5$ & & & & & \\
\hline $0 \cdot 9$ & 84 & $4 \cdot 45$ & $9 \cdot 5$ & $90 \cdot 5$ & & & & & \\
\hline $1 \cdot 0$ & 68 & $4 \cdot 56$ & $11 \cdot 3$ & $88 \cdot 7$ & & & & & \\
\hline
\end{tabular}

${ }^{a}$ All the decays were found to fit adequately by bi-exponential functions of time with $\chi^{2}$ varying between 0.97 and 1.01

almost insensitive to the alcohol concentration in the mole fraction range $0 \cdot 10-1 \cdot 0$. At mole fractions less than $0 \cdot 10$, there is a weak dependence of $k^{\mathrm{rad}}$ on alcohol concentration which is originating from the similar alcohol concentration dependence of quantum yield.

In order to investigate the effects of microheterogeneity and structural transition, the alcohol mole fraction dependence of emission transition moment for $\mathrm{C} 153$ in aqueous solutions of ethanol and TBA has also been studied. Emission transition moments $(M)$ for $\mathrm{C} 153$ have been determined from the radiative rate $\left(k^{\mathrm{rad}}\right)$ data by using the following relation $^{61(\mathrm{a}, \mathrm{b})}$

$$
M / D=1785.7\left(\frac{k^{\mathrm{rad}} / s^{-1}}{n_{s}^{3}\left(\tilde{v}^{3} / \mathrm{cm}^{-3}\right)}\right)^{1 / 2},
$$

where $\tilde{v}^{3}=\int F(v) \mathrm{d} v / \int F(v) v^{-3} \mathrm{~d} v$ with $F(v)$ denoting the fluorescence emission spectrum of C153 in alcohol-water mixture at any given mole fraction. Note that the number (1785.7) in (4) is a dimensionless quantity, a full form of which is available in ref $61(\mathrm{~b})$. The results obtained by using (4) are shown in the bottom panel of figure 3. The maximum error bar associated with this calculation is $\pm 15 \%$. The data in this figure (figure 3 ) suggest that at alcohol mole fractions $<0 \cdot 10$, there seems to be a small decrease in emission transition moment of C153 in both ethanol-water and TBA-water mixtures. The fact that the alcohol induced modification of the $\mathrm{H}$ bonding structure has small but non-negligible effects on the emission transition moment is also seen in our earlier studies with a different probe molecule in TBA-water mixture. ${ }^{65}$ However, once the tetrahedral-like H-bonding network structure of water is disrupted and chain-like structure of alcohol appears at higher alcohol concentration, the emission transition moment becomes almost insensitive to the alcohol mole fraction (between $0 \cdot 10$ and 1.0). The near insensitivity of the emission transition moment on the average polarity of the medium has also been observed earlier with a different probe in neat solvents $^{61}$ and also in electrolyte solutions. ${ }^{59}$ All these observations indicate that the solution structure rather than the polarity of the medium possibly dictates the emission transition moment in solution phase.

\subsection{Time resolved fluorescence emission studies}

As already mentioned in $\$ 2$, emission decays of C 153 in ethanol-water and TBA-water solutions have been found to be bi-exponential with time at all TBA mole fractions. The short and long time constants obtained from the fit of the magic angle emission decays probably associate with the fast solvent reorganization (spectral relaxation) and life-time of the excited probe molecule. This is rather interesting 
because in water-TBA mixtures where microscopic heterogeneity governs the solution structure, a stretched exponential rather than a simple bi-exponential function is expected to properly describe the emission decay. A closer look at the fit parameters given in table 2 reveals that short time constants are differing at all alcohol concentrations for both the ethanolwater and TBA-water mixtures, whereas the long time constants are closer to each other. Note that the ratio between the short time constants for pure TBA and ethanol is $\sim 3$ and is close to the ratio between the viscosities of these two solvents $\left(\eta_{\mathrm{TBA}} / \eta_{\text {ethanol }} \approx 4\right) .{ }^{10,11}$ This indicates that the short time is associated with the solvent reorganization. Also note that the long time

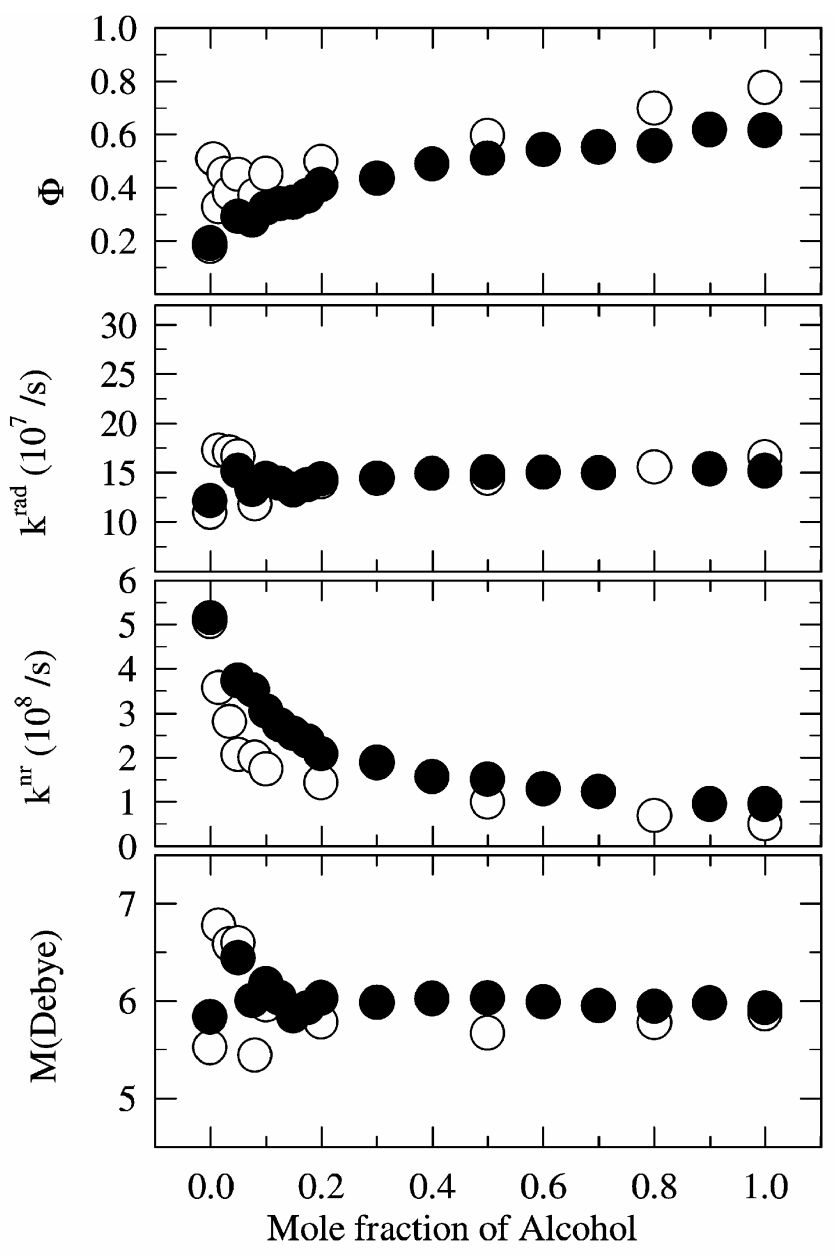

Figure 3. Alcohol mole fraction dependence of quantum yield ( $\phi$, upper panel), radiative rate $\left(k^{\text {rad }}\right.$, first middle panel), non-radiative rate $\left(k^{n r}\right.$, second middle panel) and emission transition moment $(\mathrm{M}$, bottom panel) for C153 in TBA-water and ethanol-water mixtures. While the open symbols represent the data for TBA-water mixtures, the filled symbols denote those for ethanol-water solutions. constants for the TBA-water mixtures are systematically larger than those for ethanol-water mixtures at all alcohol mole fractions, even though the dielectric constant of ethanol is higher than that of TBA. Since the quantum yields and radiative rates for C153 are comparable for these mixtures (see figure 3), the smaller non-radiative rates in TBA-water mixtures than in ethanol-water solutions lead to longer excited state life-time of $\mathrm{C} 153$ in otherwise less polar solvent TBA. This might be possible because bulkier TBA molecules would cause less collisional deactivation than the relatively lighter ethanol molecule.

Since the coupling of the solute rotation with the immediate environment controls the rotational diffusion of a solute, time resolved fluorescence anisotropy studies have been used extensively to understand the nature of the solute-environment interaction and the coupling of the solute rotation with the dynamical solvent modes. ${ }^{62}$ The time scale of the solvent modes, on the other hand, are governed by several factors, such as average polarity, structural correlations (and $\mathrm{H}$-bonding network for associating liquids), heterogeneity in the environment, preferential solvation and inter-diffusion. Therefore, time resolved fluorescence anisotropy provides an opportunity to probe the microscopic heterogeneity of alcoholwater binary mixtures and the associated structural transition.

Representative bi-exponential fit to the constructed time resolved anisotropy from the collected parallel and perpendicular emission decays for $\mathrm{C} 153$ in TBA-water solutions at 0.80 TBA mole fraction is shown in figure 4 . The nature of the residual (bottom panel) and the fit parameters listed in the upper panel indicate that a bi-exponential function is indeed required for adequate description of the $r(t)$. Except at extremely dilute ethanol concentrations, bi-exponential fits of similar quality have been obtained for all ethanol-water and TBA-water solutions studied here. Even though the single exponential fits to the $r(t)$ decays at very low ethanol mole fractions (up to $0 \cdot 10$ ) have generated reasonable values for the goodness of fit parameter $\left(\chi^{2}\right)$, the residuals (not shown here) seem to indicate a presence of a second component. It is to be noted here that earlier studies of rotational anisotropy with different solute molecules in TBA-water mixtures have reported single exponential decay of $r(t)$ with time at all TBA mole fractions. ${ }^{10,11}$ However, it was argued later by Maroncelli and coworkers ${ }^{62}$ that biexponential decay of $r(t)$ is rather generic in nature 
and is dictated by the time dependence of the microscopic friction governing the rotational diffusion in solution. More precisely, the non-exponential nature of the microscopic friction experienced by the rotating solute renders the multi-exponential decay of the time resolved fluorescence anisotropy of $\mathrm{C} 153$ in these solutions. ${ }^{62}$

Figure 5 depicts the alcohol mole fraction dependence of the fit parameters required to describe adequately the time resolved fluorescence anisotropies $(r(t))$ obtained for $\mathrm{C} 153$ in ethanol-water and

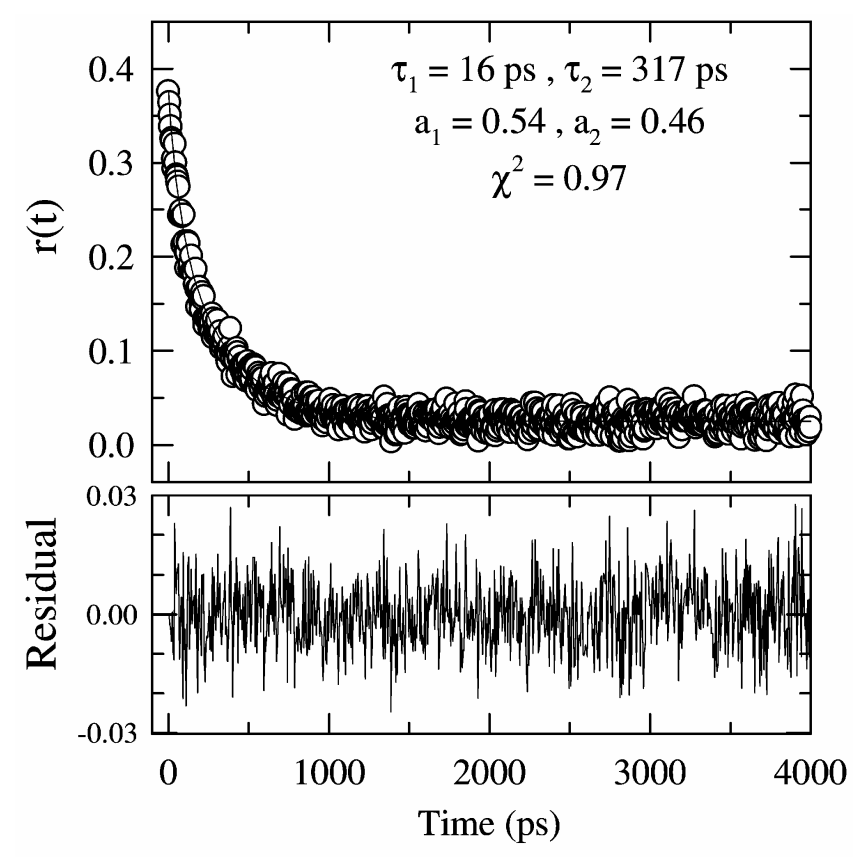

Figure 4. Representative emission anisotropy decay (upper panel) of C153 in TBA-water mixture at 0.8 TBA mole fraction. While open circles represent the data, the solid line shows the fit through the data. Parameters obtained from bi-exponential fit to the data are also listed in the upper panel. The quoted $\chi^{2}$ is actually the reduced $\chi^{2}$. Residuals (weighted) are shown in the bottom panel. Note that the fast time constant $(\sim 15 \mathrm{ps})$ is one-fifth of the FWHM of the IRF employed in our experiments and hence is at the detection limit. The standard error of estimate for the shorter time scale ( $\tau_{\text {short }}$ ) is about $30 \%$ of the quoted value at each alcohol mole fraction (due to limited time resolution) and the same for the long time scale ( $\tau_{\text {long }}$ ) is about $10 \%$ (of the quoted value). The standard error of estimate for the amplitudes, however, is approximately $5 \%$ (of the quoted value) at each alcohol mole fraction. Note here that the definitions for the standard error as well as those for reduced $\chi^{2}$ and weighted residual are used as those described in the following reference: Bevington P R Data Reduction and Error Analysis for the Physical Sciences, McGraw-Hill: New York, 1969.
TBA-water solutions. While the time constants are shown in the left panels of this figure, the right panels describe the alcohol concentration dependence of the amplitudes associated with the $r(t)$ decays. It is interesting to note in this figure that the long time constant ( $\left.\tau_{\text {long }}\right)$ and the amplitude associated with it $\left(a_{\text {long }}\right)$ increase initially and then saturate. The initial increase is particularly very sharp for TBA-water mixtures. The short time constant $\left(\tau_{\text {short }}\right)$ and the corresponding amplitude $\left(a_{\text {shorr }}\right)$, on the other hand, decrease upon increasing the alcohol mole fraction in the mixtures. The large difference between $\tau_{\text {short }}$ and $\tau_{\text {long }}$ may lead one to think that the bi-exponential decay of $r(t)$ is originating from the rotational diffusion of C153 trapped in two different solvation environments - one which is predominantly enriched with alcohol molecules and the other with water molecules. Support for such a two state model for the solution structure of alcohol-water mixtures has also been provided earlier by light scattering studies. ${ }^{18}$ As the tetrahedral network structure of water is strengthened upon addition of small quantity of alcohol in the water-rich region, the solution structure becomes more compact. The increased compactness

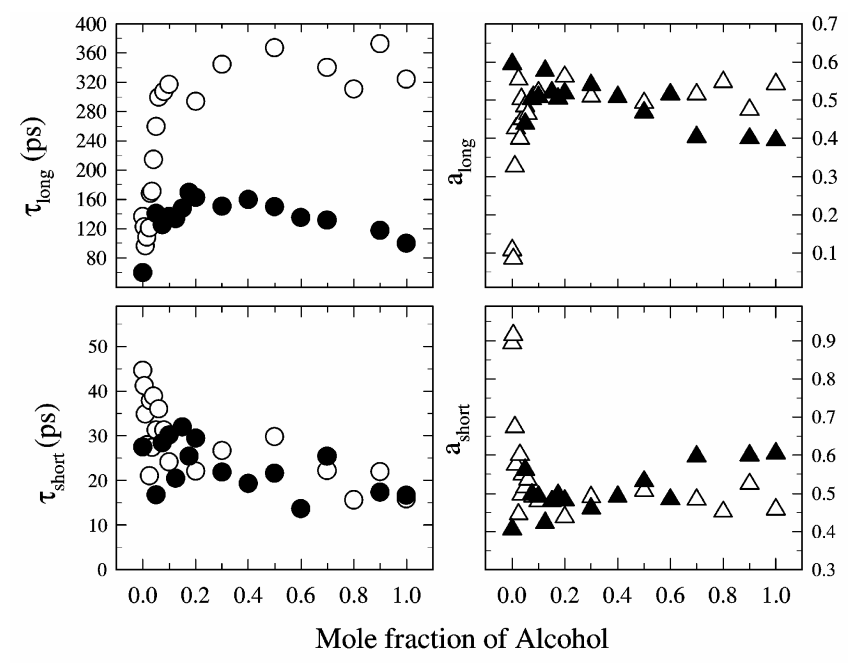

Figure 5. Alcohol mole fraction dependence of fit parameters required to adequately describe the experimental time resolved fluorescence anisotropy of C153 in TBAwater (open symbol) and ethanol-water (filled symbol) mixtures. Note that the time constants are shown in the left panels and the amplitudes in the right panels. The faster time constants obtained from the bi-exponential fits are represented by $\tau_{\text {short }}$ and the slower ones by $\tau_{\text {long. }}$. The corresponding amplitudes are represented respectively by $a_{\text {short }}$ and $a_{\text {long. }}$. The goodness of fit parameter (reduced $\chi^{2}$ ) for all these fits varies between 0.97 and 1.03. The standard errors of estimate are already described in figure 4 . 
of solution structure in the water rich region is also reflected in the lowering of isothermal compressibility ${ }^{38}$ in the corresponding alcohol concentration range. The increase in structural compactness with alcohol concentration therefore leads to further slowing down of the long time constant with concomitant increase in the amplitude of this component. Further addition of alcohol induces a transition in the solution structure from the tetrahedral network to zigzag chain-like molecular contacts giving rise to relatively more microscopically 'homogeneous' environment. This 'homogeneity' in the environment leads to the saturation of both the long time component and its amplitude at higher alcohol mole fraction.

The next question is why the short time constant ( $\left.\tau_{\text {short }}\right)$ becomes smaller as the alcohol concentration is increased in the low mole fraction regime of alcohol in the mixture? This can be explained if we look at the mode coupling theory (MCT) analyses of density dependent microscopic friction on a translating solute in liquids. ${ }^{66}$ According to the MCT, the enhanced solute-solvent collision frequency due to stronger structure at higher density leads to faster decay of time dependent friction on the solute. ${ }^{66}$ As the same structural features also enhance the viscosity of the medium due to stronger spatial correlations, the viscosity driven long-time decay of the microscopic friction becomes slower at later times. ${ }^{66}$ Therefore, the structural enhancement makes the fast component faster and the slow component slower. This is probably the reason why the short time constant $\left(\tau_{\text {short }}\right)$ in the bi-exponential decay of $r(t)$ for C153 in alcohol-water mixtures becomes smaller

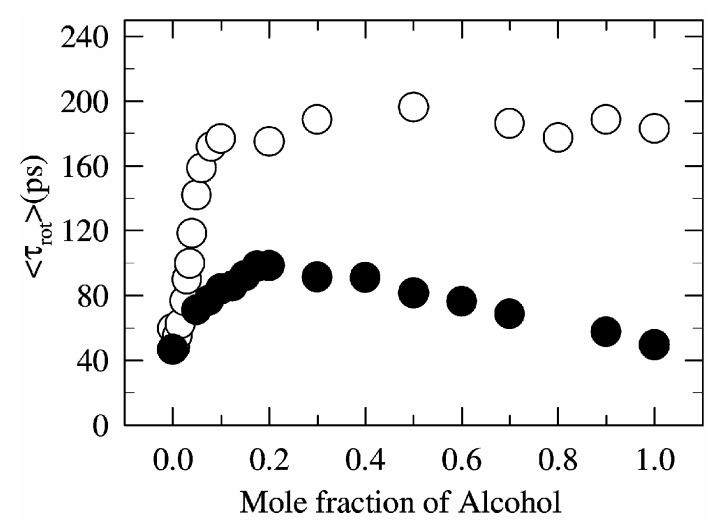

Figure 6. Alcohol mole fraction dependence of average rotational time $\left(\left\langle\tau_{\text {rot }}\right\rangle\right)$ for C153 in TBA-water and ethanol-water mixtures. While the open circles represent the data for TBA-water mixtures, filled circles denote those for ethanol-water mixtures. with alcohol mole fraction at very low alcohol concentration.

The long time constant $\left(\tau_{\text {long }}\right)$ for ethanol-water mixture, however, shows a moderate decrease with increase in alcohol concentration at ethanol mole fraction $>0 \cdot 20$. Since ethanol and water are nearly isoviscous solvents, the loss of water structure at higher ethanol concentration dominates the long time decay constant and $\tau_{\text {long }}$ shows a decrease in this ethanol mole fraction range. For TBA-water mixture, on the other hand, the effects of viscosity rather than water structure controls the dynamics as the viscosity of TBA is approximately 5 times larger than that of water. ${ }^{10,11}$ As a result, $\tau_{\text {long }}$ in presence of TBA nearly saturates at higher alcohol mole fraction, whereas in presence of ethanol it shows a decrease with increasing ethanol mole fraction.

Figure 6 depicts the alcohol mole fraction dependence of average rotational correlation time, $\left\langle\tau_{\mathrm{rot}}\right\rangle$ for $\mathrm{C} 153$ in ethanol-water and TBA-water mixtures. Note that these times $\left(\left\langle\tau_{\text {rot }}\right\rangle\right)$ have been obtained from the fit parameters described in figure 5 . The value of the $\left\langle\tau_{\text {rot }}\right\rangle$ for $\mathrm{C} 153$ in pure ethanol shown in this figure 6 compares well with the literature value. $^{62}$ The most interesting aspect of this figure is the difference in slope of $\left\langle\tau_{\text {rot }}\right\rangle$ with alcohol mole fraction for ethanol-water and TBA-water mixtures. The stronger hydrophobic effect of TBA due to its bulkier tertiary butyl group is responsible for the increased orientational ordering (structure formation) of the water molecules leading to a sharp increase in the $\left\langle\tau_{\text {rot }}\right\rangle$ for $\mathrm{C} 153$ in TBA-water mixture at very dilute TBA concentration. The sharp slope then transforms to an almost flat line at higher TBA concentration and this transformation occurs at a TBA mole fraction $\sim 0 \cdot 10$. The slope for ethanol-water mixtures at low ethanol concentration is not as sharp because of less structure forming ability of ethanol molecules. Nevertheless, a change in slope with alcohol mole fraction is also visible here which occurs at ethanol mole fraction $\sim 0 \cdot 2$. These values of alcohol mole fraction at which the slope changes, corroborate well with the literature values where structural transition is believed to occur in these alcohol-water mixtures.

\section{Conclusion}

In this paper, we have studied the effects of solution structure on the steady state spectroscopic properties of $\mathrm{C} 153$ and its rotational dynamics in ethanol- 
Table 3. Comparison among time resolved data reported by this work and other groups.

\begin{tabular}{|c|c|c|c|c|c|c|}
\hline$\lambda_{\mathrm{exc}}(\mathrm{nm})$ & IRF (ps) & Method & $\begin{array}{c}\text { No. of } \\
\text { exp. functions }{ }^{a}\end{array}$ & $\tau_{\text {fast }}(\mathrm{ps})^{\mathrm{b}}$ & $\tau_{\text {slow }}(\mathrm{ps})$ & $\tau_{\mathrm{L}}(\mathrm{ps})^{\mathrm{c}}$ \\
\hline 405 (ref. 67) & 110 & TCSPC & 1 & - & - & 4680 \\
\hline 387 (ref. 62) & $0 \cdot 120$ & Up-conversion & 2 & 10.4 & 89 & - \\
\hline 420 (ref. 68) & $0 \cdot 120$ & Up-conversion & 2 & 14 & 92 & 4850 \\
\hline 409 (present work) & 75 & TCSPC & 2 & 16 & 99 & 4560 \\
\hline \multicolumn{7}{|c|}{$\begin{array}{l}\text { Number of exponentials required to adequately fit the anisotropy decay for C153 in ethanol } \\
\mathrm{b} \\
{ }^{\mathrm{b}} \tau_{\text {fast }} \text { and } \tau_{\text {slow }} \text { are two time constants associated with the bi-exponential decay of anisotropy (C153 in } \\
\text { ethanol) } \\
{ }^{\mathrm{c}} \tau_{\mathrm{L}} \text { represents the longest time constant associated with the multi-exponential magic angle decay of C153 } \\
\text { in ethanol collected at or nearly at the wavelength corresponding to the peak of the steady state emission } \\
\text { spectrum. Ref. } 55 \text { have shown (figure } 4, \mathrm{p} .17316 \text { ) that the magic angle decay of C153 in methanol col- } \\
\text { lected at } 480 \mathrm{~nm} \text { requires } 4 \text {-exponentials with } 3900 \mathrm{ps} \text { as } \tau_{\mathrm{L}} \text { (obtained via TCSPC) in order to adequately } \\
\text { fit the decay. } \lambda_{\text {exc }} \text { represents the excitation wavelength and IRF the full width at half maximum of the in- } \\
\text { strument response function }\end{array}$} \\
\hline
\end{tabular}

water and TBA-water mixtures. The enhancement of solution structure is manifested in the red-shift of the absorption spectrum of $\mathrm{C} 153$ at very dilute alcohol concentration. The emission peak frequencies, however, do not reflect the effects of such structural enhancement as the average excited state life time of C153 is much larger than the time scales on which the alcohol clusters are stable. Quantum yield is found to increase slightly with alcohol mole fraction in the alcohol concentration range $0 \cdot 10-1 \cdot 0$ in these mixtures, whereas both the radiative rate and emission transition moment show near insensitivity to the alcohol concentration in the same range.

The decay of the time resolved fluorescence anisotropy has been found to be bi-exponential in ethanol-water and TBA-water mixtures at all alcohol mole fractions. Structural effects have been found to play a significant role in determining the mole fraction dependence of the time constants as well as the amplitudes associated with the decay components of $r(t)$ in these binary mixtures. The sharp increase in the average rotational correlation time with alcohol concentration in the very dilute solution is explained in terms of structural modifications due to hydrophobic effects of alkyl groups attached to these alcohols. The novel interplay between solution structure and viscosity critically determines the effects of structural transition on the rotational motion of C153 in these alcohol-water mixtures. The magic angle emission decay is also found to be bi-exponential at all alcohol mole fractions where relatively lower non-radiative rate in TBA-water mixture is found to be responsible for longer excited state life time of $\mathrm{C} 153$ than in ethanol-water mixture.
An important aspect to be mentioned here is that any impurity in the probe (C153) could also lead to the multi-exponential decay of the time resolved anisotropy in these mixtures. We have examined this possibility by comparing our both steady state absorption and emission, and time resolved data with those from several groups. For example, the steady state absorption and emission peak frequencies of C153 in ethanol respectively are (in the unit of $10^{3} \mathrm{~cm}^{-1}$ ) 24 and $18 \cdot 20$, which compare well those (24.08 and 18.10) from Ref. 55. The corresponding bandwidths (FWHM) are (in the unit of $10^{3} \mathrm{~cm}^{-1}$ ) 4.02 and 3.52 and also agree with the literature values (3.88 and 3.18) ${ }^{55}$ Time resolved data from various groups ${ }^{62,67,68}$ are summarized and compared in table 3. It is clear from this table that both the fast and slow time constants ( $\tau_{\text {fast }}$ and $\left.\tau_{\text {slow }}\right)$ are agreeing well with the data from other research groups ${ }^{62,68}$ which used much better time resolution (120 fs) compared to ours $(\sim 75 \mathrm{ps})$. Also, the longest time constant $\left(\tau_{\mathrm{L}}\right)$ of the multi-exponential decay of emission intensity collected at magic angle $\left(54.7^{\circ}\right)$ compares well with the values reported by these groups. ${ }^{62,67,68}$ All these evidences support the conclusion that the observed bi-exponential decay of the anisotropy of $\mathrm{C} 153$ in these binary mixtures is rather generic in nature and might therefore not be associated with any impurity in the probe or misalignment in the measurement system. Also note that the shortest life time obtained from the fit of the magic angle decay for C153 in ethanol is $\sim 70$ ps (table 2) which is at least 4 times greater than the fastest component in the anisotropy decay (figure 5, left lower panel, filled circles). Such a difference is believed to be 
large enough for the anisotropy decay to be completely decoupled from the fast solvent relaxations.

The effects of solution structure and structural transition could be studied in binary mixtures of polar and non-polar components as well as in polarquadrupolar mixtures. As several dynamical processes such as vibrational energy and phase relaxations, decay of force-force correlation functions controlling the friction on a rotating moiety involved in a chemical reaction and diffusion processes are governed by the coupling of the solvent structure to the reactive motion, ${ }^{69}$ understanding the solutesolvent interactions in such binary mixtures are important. Study of the effects of a third component, which could be a non-polar solute or simply an electrolyte, on this structural modifications and the related consequences on various dynamical processes in solution mixtures could also constitute an interesting problem. Some of the studies discussed above are already in progress. ${ }^{70}$

\section{Acknowledgements}

We thank the Director, SNBNCBS for support and encouragement. Partial financial assistances from the Department of Science and Technology (DST), and Council of Scientific and Industrial Research (CSIR), India are gratefully acknowledged. T P thanks University Grants Commission (UGC) for a research fellowship. We thank an anonymous referee for several helpful suggestions.

\section{References}

1. Franks F and Ives D J G 1966 Q. Rev. Chem. Soc. 201

2. Zeidler M D 1973 In Water, a comprehensive treatise (ed.) F Franks (New York: Plenum), vol 2, p 529

3. Arnett E M and McKlevey D R $1966 \mathrm{~J}$. Am. Chem. Soc. 885031

4. Arnett E M and McKlevey D R $1965 \mathrm{~J}$. Am. Chem. Soc. 871393

5. Arnett E M, Bentrude W G, Burke J J and Duggleby P M 1965 J. Am. Chem. Soc. 871541

6. Beddard G S, Doust T and Hudales J 1981 Nature 294145

7. Dixit S, Crain J, Poon W C K, Finney J L and Soper A K 2002 Nature 416829

8. Soper A K and Finney J L 1993 Phys. Rev. Lett. 71 4346

9. Brai M and Kaatze U 1992 J. Phys. Chem. 908946

10. Dutt G B and Doraiswamy S 1992 J. Chem. Phys. 96 2475

11. Dutt G B, Doraiswamy S and Periasamy N $1991 \mathrm{~J}$. Chem. Phys. 942475
12. Murthy S S N 1999 J. Phys. Chem. A103 7927

13. Wojkow D and Czarnecki M A 2005 J. Phys. Chem. A109 8218

14. Iwasaki K and Fujiyama T 1979 J. Phys. Chem. 83 463; Iwasaki K and Fujiyama T 1977 J. Phys. Chem. 811908

15. D'Angelo M, Onori G and Santucci A $1994 \mathrm{~J}$. Chem. Phys. 1003107

16. Egashira K and Nishi N 1998 J. Phys. Chem. B102 4054

17. Nishikawa K and Iijima T 1993 J. Phys. Chem. 97 10824

18. Yoshida K and Yamaguchi T 2001 Z. Naturforsch. A56 529

19. Nishikawa K, Kodera Y and Iijima T 1987 J. Phys. Chem. 913694

20. Koga Y 1984 Chem. Phys. Lett. 111176

21. Sato T and Buchner R 2003 J. Chem. Phys. 119 10789

22. Nakanishi K, Ikari K, Okazaki S and Touhara H 1984 J. Chem. Phys. 801656

23. Kusalik P G, Lyubertsev A P, Bergman D L and Laaksonen A 2000 J. Phys. Chem. B104 9533

24. Laaksonen A, Kusalik P G and Svishchev I M $1997 \mathrm{~J}$. Phys. Chem. A101 5910

25. Yoshida K, Yamaguchi T, Kovalenko A and Hirata $\mathrm{F}$ 2002 J. Phys. Chem. B106 5042

26. Perera A, Sokolic F, Almasy L and Koga Y $2006 J$. Chem. Phys. 124124515

27. Ben-Naim A 1977 J. Chem. Phys. 674884

28. Bowron D T and Finney J L 2007 J. Phys. Chem. B111 9838

29. Lisa L, Loon V, Minor R N and Allen H C $2007 \mathrm{~J}$. Phys. Chem. A111 7346

30. Hu K, Zhou Y, Shen J, Ji Z and Cheng G $2007 \mathrm{~J}$. Phys. Chem. B111 10160

31. Templeton E F G and Kenney-Wallace G A $1986 \mathrm{~J}$. Phys. Chem. 905441

32. Iwasaki K and Fujiyama T 1977 J. Phys. Chem. 81 1908

33. Iwasaki K and Fujiyama T 1979 J. Phys. Chem. 83 463

34. Euliss G W and Sorensen C M 1984 J. Chem. Phys. 804767

35. Kaatze U, Pottel R and Schmidt $\mathrm{P} 1988$ J. Phys. Chem. 92 3669; Kaatze U, Pottel R and Schmidt P 1989 J. Phys. Chem. 935623

36. Kaatze U, Menzel K and Pottel R 1991 J. Phys. Chem. 95324

37. Svishchev I M and Kusalik, P G 1993 J. Chem. Phys. 993049

38. Omelyan I, Kovalenko A and Hirata F 2003 J. Theor. Comput. Chem. 2193

39. Chandra A and Bagchi B 1991 J. Chem. Phys. 94 8367

40. Bagchi B and Biswas R 1999 Adv. Chem. Phys. 109 207

41. Cichos F, Willert A, Rempel U and von Borczyskowski C 1997 J. Phys. Chem. A101 8179

42. Luther B M, Kimmel J R and Levinger N E $2002 \mathrm{~J}$. Chem. Phys. 1163370 
43. Chandra A 1995 Chem. Phys. Lett. 235133

44. Jarzeba W, Walker G C, Johnson A E and Barbara P F 1991 Chem. Phys. 15257

45. Gardecki J A and Maroncelli M 1999 Chem. Phys. Lett. 301571

46. Ladanyi B M and Skaf M S 1996 J. Phys. Chem. A100 18258

47. Laria D and Skaf M S 1999 J. Chem. Phys. 111300

48. Day T J F and Patey G N 1997 J. Chem. Phys. 1062782

49. Day T J F and Patey G N 1999 J. Chem. Phys. 110 10937

50. Yoshimori A, Day T J F and Patey G N $1998 \mathrm{~J}$. Chem. Phys. 1086378

51. Yoshimori A, Day T J F and Patey G N $1998 \mathrm{~J}$. Chem. Phys. 1093222

52. Bowron D T and Moreno S D 2005 J. Phys. Chem. B109 16210

53. Kashyap H K and Biswas R 2007 J. Chem. Phys. 127 184502

54. Kashyap H K and Biswas R 2007 J. Chem. Sci. 119 391

55. Horng M L, Gardecki J A, Papazyan A and Maroncelli M 1995 J. Phys. Chem. B99 17311

56. Reynolds L, Gardecki J, Frankland S J, Horng M L and Maroncelli M 1996 J. Phys. Chem. 10010337

57. Biswas R, Lewis J E and Maroncelli M 1999 Chem. Phys. Lett. 310485
58. Lewis J E, Biswas, R, Robinson A G and Maroncelli M 2001 J. Phys. Chem. B105 3306

59. Pradhan T and Biswas R 2007 J. Phys. Chem. A111 11514

60. Pradhan T and Biswas R 2007 J. Phys. Chem. A111 11524

61. (a) Dahl K, Biswas R, Ito N and Maroncelli M 2005 J. Phys. Chem. B109 1563; (b) Lewis J E and Maroncelli M 1998 Chem. Phys. Lett. 282197

62. Horng M L, Gardecki J A and Maroncelli M $1997 \mathrm{~J}$. Phys. Chem. A101 1030

63. Nishi N and Yamamoto K $1987 \mathrm{~J}$. Am. Chem. Soc. 1097353

64. Nishi N, Koga K, Ohsima C, Yamamoto K, Nagashima U and Nagami K $1988 \mathrm{~J}$. Am. Chem. Soc. 110 5246

65. Pradhan T, Ghoshal P and Biswas R 2008 J. Phys. Chem. A 112915

66. Bagchi B and Bhattacharyya S 2001 Adv. Chem. Phys. 11667

67. Maroncelli M and Fleming G R 1987 J. Chem. Phys. 866221

68. Baumann R, Ferrante C, Kneuper E, Deeg F-W and Brauchle C 2003 J. Phys. Chem. A107 2422

69. Biswas R and Bagchi B 1996 J. Chem. Phys. 105 207

70. Biswas R unpublished results 